\title{
Structure-Based Virtual Screening for the Discovery of Novel Inhibitors of New Delhi Metallo- $\beta$-lactamase-1
}

Francesca Spyrakis, ${ }^{\dagger, \perp \odot}$ Giuseppe Celenza, ${ }^{\ddagger}$ Francesca Marcoccia, ${ }^{\ddagger}$ Matteo Santucci, ${ }^{\dagger}$ Simon Cross, $^{\S}$ Pierangelo Bellio, ${ }^{\ddagger}$ Laura Cendron, $\|$ Mariagrazia Perilli, ${ }^{\ddagger}$ and Donatella Tondi* ${ }^{\dagger} \dagger$

${ }^{\dagger}$ Dipartimento di Scienze della Vita, Università degli Studi di Modena e Reggio Emilia, Via Campi 103, 41100 Modena, Italy

${ }^{\ddagger}$ Dipartimento di Scienze cliniche applicate e biotecnologiche, Università dell'Aquila, Via Vetoio 1, 67100 L’Aquila, Italy

${ }^{\S}$ Dipartimento di Chimica, Biologia e Biotechnologia, Università degli Studi di Perugia, Via Elce di Sotto 8, 06123 Perugia, Italy

"Dipartimento di Biologia, Università degli Studi di Padova, Viale G. Colombo 3, 35131 Padova, Italy

\author{
Supporting Information
}

ABSTRACT: Bacterial resistance has become a worldwide concern after the emergence of metallo- $\beta$-lactamases (MBLs). They represent one of the major mechanisms of bacterial resistance against beta-lactam antibiotics. Among MBLs, New Delhi metallo- $\beta$-lactamase- 1 NDM-1, the most prevalent type, is extremely efficient in inactivating nearly all-available antibiotics including last resort carbapenems. No inhibitors for NDM-1 are currently available in therapy, making the spread of NDM-1 producing bacterial strains a serious menace. With this perspective, we performed a structure-based in silico screening of a commercially available library using FLAPdock and identified several, non- $\beta$-lactam derivatives as promising candidates active against NDM-1. The binding affinities of the highest scoring hits were measured in vitro revealing, for some of them, low micromolar affinity toward NDM-1. For the best inhibitors, efficacy against resistant bacterial strains overexpressing NDM-1 was validated, confirming their favorable synergistic effect in combination with the carbapenem Meropenem.

KEYWORDS: NDM-1 Metallo- $\beta$-Lactamase, carbapenemases, bacterial resistance, structure-based virtual screening, enzyme inhibition, 4H-1,2,4-triazole-3-thiol derivatives

$\mathrm{T}^{\mathrm{k}}$ he extensive use of antibiotics has created major resistance problems leading to increased morbidity, mortality, and healthcare costs. ${ }^{1}$ Among several mechanisms of resistance pathogenic bacteria have developed against antimicrobial therapy, the hydrolysis of $\beta$-lactam antibiotics by $\beta$-lactamase enzymes is one of the most prevalent in resistant strains. ${ }^{2,3}$ BLs have emerged in Gram-positive and -negative bacteria and evolved in extended spectrum $\beta$-lactamases (ESBLs) first and carbapenemases later, leaving not many alternatives to microbial infections. ${ }^{2-5}$ BLs belong to two main groups: serine-based BLs (SBLs class A, C, D) and metallo-BLs (class B). ${ }^{6}$ With respect to SBLs, for which approved inhibitors are available in combination with beta-lactam antibiotics ${ }^{7}$ and few others are in the development pipeline, ${ }^{8}$ at present no drug capable of inhibiting any of the class B zinc-dependent metallo- $\beta$-lactamases is available in therapy.

MBLs use a zinc-bound hydroxyl group as nucleophile to catalyze the hydrolysis of a very broad range of $\beta$-lactam antibiotics including penicillins, cephalosporins, carbapenems, and $\mathrm{BL}$ inhibitors, with the only exception of monobactams. ${ }^{5}$ Therefore, the emergence of these enzymes is particularly concerning for the future treatment of bacterial infections.

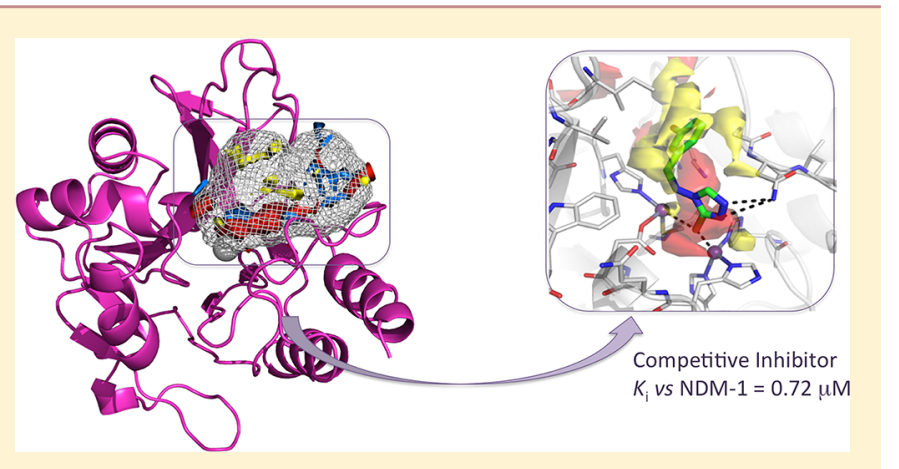

Among MBLs, the clinically relevant metallo-carbapenemase NDM-1 (New Delhi metallo- $\beta$-lactamase) is the most worrisome in light of its substrate promiscuity, its resistance to nearly all available drugs, and its easiness of variants appearance and transferability. NDM-1 belongs to the B1 family of metallo- $\beta$ lactamases, and since its discovery in bacterial infections harboring plasmid-encoded NDM-1, this carbapenemase has emerged as a global health threat. ${ }^{9}$ It has caused multiple epidemics and it is capable of easy propagation to other species. ${ }^{10}$ Moreover, most plasmids harboring the NDM-1 gene are often associated with other resistance markers, making NDM-1 positive strains resistant to multiple drugs. ${ }^{10-12}$

As a consequence, there is a growing need for novel inhibitors against NDM-1, targeted in this study.

To identify novel MBLs inhibitors, starting from the available NDM-1 crystal structures [PDB code $3 \mathrm{q} 6 \mathrm{x}$ and $3 \mathrm{spu}$ ], a structure-based virtual screening (SBVS) of a large database of available chemicals was conducted, searching for potential inhibitors able to highly complement NDM-1 binding site. For

Received: October 17, 2017

Accepted: November 25, 2017

Published: November 26, 2017 
Table 1. $K_{\mathrm{i}}$ Values for the Most Representative NDM-1 Inhibitors

\begin{tabular}{|c|c|c|c|c|c|c|c|c|}
\hline Cpd & Structure & $K_{\mathrm{i}}(\mu \mathrm{M})^{[\mathrm{a}]}$ & Cpd & Structure & $K_{\mathrm{i}}(\mu \mathrm{M})^{[\mathrm{a}]}$ & Cpd & Structure & $K_{\mathrm{i}}(\mu \mathrm{M})^{[\mathrm{a}]}$ \\
\hline 1 & & $\begin{array}{l}0.72 \\
\pm 0.014\end{array}$ & 7 & & $\begin{array}{l}65 \\
\pm 1.35\end{array}$ & 11 & & $\begin{array}{l}71 \\
\pm 2.0\end{array}$ \\
\hline 2 & & $\begin{array}{l}1.00 \\
\pm 0.022\end{array}$ & 8 & & $\begin{array}{l}67 \\
\pm 2.6\end{array}$ & 12 & & $\begin{array}{l}76 \\
\pm 1.5\end{array}$ \\
\hline 3 & & $\begin{array}{l}52 \\
\pm 1.3\end{array}$ & 9 & & $\begin{array}{l}68 \\
\pm 1.9\end{array}$ & 15 & & $\begin{array}{l}85 \\
\pm 3.8\end{array}$ \\
\hline
\end{tabular}

${ }^{a}$ Estimated $K_{\mathrm{i}}$ as per competitive inhibitor. ${ }^{15}$ Assays were conducted vs NDM-1 (0.5 nM), 10 min incubation, at $25{ }^{\circ} \mathrm{C}$, inhibitor concentration ranging from 0 to $500 \mu \mathrm{M}$ in HEPES $20 \mathrm{mM}(\mathrm{pH} 7.0)+20 \mu \mathrm{M} \mathrm{ZnCl}_{2}$, using Nitrocefin $30 \mu \mathrm{M}$ as reporter substrate. All the experiments were performed in triplicate.

the best inhibitors biological assays against clinical strains overexpressing NDM-1 were conducted, confirming their ability to synergize last generation antibiotics and to restore susceptibility in resistant strains.

The virtual screening was performed with FLAPdock implemented within FLAP (Fingerprints for Ligands and Proteins). ${ }^{13}$ A specific version of FLAPdock was developed ad hoc to deal with MBL's active site. ${ }^{14}$

Starting from an initial library of $\sim 300,000$ Specs commercially available compounds (www.specs.net) opportunely filtered for LogP lower than 2.5, a library of 100 compounds was selected according to the score assigned by the native scoring function, the number of hydrogen bonds formed within the binding site, their chemical diversity, and their ability to complement the NDM-1 active site. The compounds were first rapidly screened in vitro for inhibitory activity against NDM-1 through a spectrophotometric assay. Based on the determined percentage of inhibition against NDM-1 (cut off \% inhibition <50 at 200 $\mu \mathrm{M}$ ) a more focused library of 31 derivatives was selected (Table S1). Each compound was further validated for its capability to inhibit NDM-1: binding affinities and mechanism of inhibition were determined. In inhibition assays, the activity of NDM-1 was monitored following the hydrolysis rate of the substrate nitrocefin at $\lambda=480 \mathrm{~nm}$, after $10 \mathrm{~min}$ incubation of each compound with the enzymes.

We noted that the 31 active candidates are characterized by the presence of electron donor chemotype able to coordinate the zinc ions, that is, triazol-thiole, amino-triazole, tetrazole, carboxylates, sulfonyl, and sulfonamide groups. ${ }^{16-18}$ Most active inhibitors in the library for which the binding mode is herein described are reported in Table 1 (for the complete list of focused library, see Table S1).

Summarizing, among the total 31 derivatives (Table S1), we identified two compounds showing $K_{\mathrm{i}}$ of 0.72 and $1 \mu \mathrm{M}$ (Table 1 , compounds 1 and 2). Other 22 compounds, exploiting diverse chemical functionalities, showed $K_{\mathrm{i}} \mathrm{s}$ between 52 and $99 \mu \mathrm{M}$. All compounds behave as competitive inhibitors with respect to reporter substrate nitrocefin. The most active compounds $\mathbf{1}$ and 2 share the common 4H-1,2,4-triazole-3-thiol core able to advantageously interact with both $\mathrm{Zn}$ ions present in the binding site. The binding poses of compounds $\mathbf{1}$ and $\mathbf{2}$ are reported in Figure 1a,b.

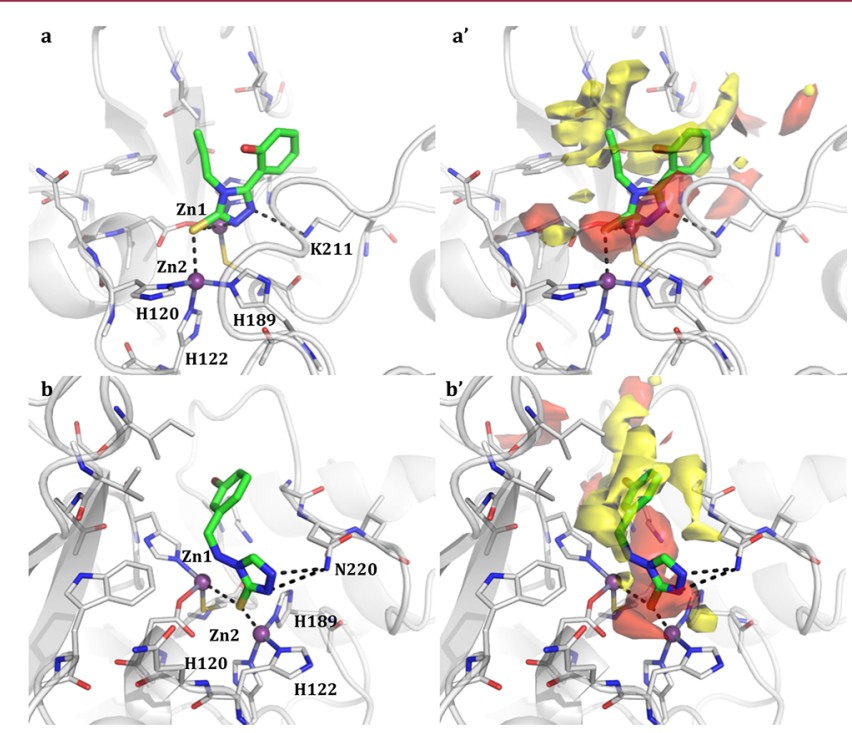

Figure 1. Binding poses of compounds 1 and 2 in NDM-1 binding site ( $\mathrm{a}$ and $\mathrm{b}$, respectively). Compounds are shown as capped sticks, zinc ions as purple spheres, and H-bonds as dashed lines. The binding site Molecular Interaction Fields are shown in panels $\mathrm{a}^{\prime}$ and $\mathrm{b}^{\prime}$. For clarity, only the hydrophobic (yellow) and hydrogen-bond acceptor (red) contours are shown. Pictures were prepared using Pymol (http://www. pymol.org).

The thiol group is a known zinc chelator and a chemical feature often shared by other reported MBL inhibitors. ${ }^{18}$ At the moment of completing this Letter, Sevaille et al. discussed the activity of ligands sharing a similar triazole-3-thiole core toward a panel of dizinc MBL, including NDM-1. ${ }^{19}$

The interactions of our compounds $\mathbf{1}$ and $\mathbf{2}$ with the zinc ions are mediated by S3 and N2, as reported for the similar 1,2,4triazole-3-thiole compound cocrystallized with L1MBL reported by Sevaille et al. ${ }^{20}$ However, in compounds $\mathbf{1}$ and 2 , the substituent on C5 assumes a different orientation given to the diverse architecture and composition of the binding site, and in particular, of the hydrophobic hot spots. Further interactions are made by N1 with Lys211, a residue normally involved in substrate recognition, orientation, and hydrolysis. This strongly supports the value of our results and the potential of the procedure, able to identify 1,2,4-triazole-3-thiole derivatives 
among the best binders in a filtered library of more than 20,000 compounds.

Compound 2 contacts both zinc ions with S3 and forms another interaction with Asn220, a key residue involved, along with Lys211, in substrate binding and in the intermediate product stabilization via formation of an oxyanion hole in conjunction with $\mathrm{Zn} 1$ (Figure $1 \mathrm{~b}$ ). Both molecules properly fit the H-bond acceptor region identified by GRID (Figure $1 a^{\prime}, b^{\prime}$ ) lined by the two ions and Lys211, and the large hydrophobic contour lined by Ile35, Val73, Trp93, and Gly219 and usually involved in hydrophobic interactions with $\beta$-lactam $\mathrm{R}$ groups.

According to the literature ${ }^{21}$ and crystallographic evidence, $^{20,22}$ thiols groups were modeled as thiolates. In the MBL binding site, sulphydryl groups exist as anions, mimicking the catalytic hydroxide, because of the zinc effect, which lowers the $\mathrm{p} K_{\mathrm{a}}$ of bound thiol groups by about two orders of magnitude. 22,23

In light of their $K_{\mathrm{i}}(0.72$ and $1 \mu \mathrm{M}$, respectively), their reversible mechanism of inhibition, their ligand efficiencies $(0.5$ $\mathrm{kcal} \mathrm{mol}^{-1}$ ), and their lead-like physical properties, they represent promising candidates for chemical derivatization. ${ }^{24}$

Compounds 5 and $\mathbf{6}$ present a triazol-thiole moiety as well, thus able to coordinate the zinc ions (Table S1). Compound 6 bears a carboxylate also involved in the coordination of one of the two ions. In the complexes formed by the other compounds, the metals are coordinated by different electron donor groups. Compounds 3 and $\mathbf{4}$ interact with the ions by means of the sulfonyl or sulfonamide moiety (see Table 1, Table S1). In particular, compound 3 contacts both of them through the carbonyl and the sulfonyl moiety, also interacting with Lys 211 and Asn220 (Figure 2a).

In the case of compound 7 (Table 1, Figure 2b), the coordination contacts are made by both the purine ring and the sulfonic acid, still forming hydrogen bonds with Lys 211 and Asn220, while for compound $\mathbf{1 0}$ only the sulfonylic amid moiety is responsible for the metal coordination (Table S1). Compounds 8 presents a tetrazol-amine moiety, with N2, N3, and N4 coordinating both $\mathrm{Zn} 1$ and $\mathrm{Zn} 2$ (Table 1, Figure 2c).

Similarly, compounds 11 (Table 1, Figure 2d) and 14 (Table S1) exploit the presence of a tetrazol ring and, also, of the sulfonyl moiety to bind the protein active site. In the case of compounds 9 (Table S1), 12 (Table 1, Figure 2e), and $\mathbf{1 3}$ (Table S1), the coordination is mediated by carboxylic functions. In particular, compound 12 (Figure 2e) is able to contact $\mathrm{Zn} 1$ and $\mathrm{Zn} 2$ with the single carboxylic acid and to further stabilize its orientation in the pocket through the formation of an additional hydrogen bond with Asn220. Compound $\mathbf{1 5}$ combines a carboxylic function and a triazol-thiole group, both involved in the metals coordination. The carboxylic moiety also contacts Asn220 (Table 1, Figure 2f). Compounds $\mathbf{1 6}$ and $\mathbf{1 7}$ are characterized by the presence of a triazole carboxamide and a triazole amine, respectively (Table $\mathrm{S} 1$ ).

Apparently, the presence of a mercapto-triazole moiety one/ two atom spaced from an aromatic ring represents a suitable scaffold to stably bind the enzyme active site and inhibit its activity. The identified compounds could be further optimized to better fit the target binding site shape and MIFs.

To verify the ability of the compounds to synergically interact with therapeutically available antibiotics, a microdilution drugdrug interaction assay was conducted both on E. coli clinical isolate expressing NDM-1 enzyme and E. coli BL21 (DE3) recombinant strain harboring the vector $\mathrm{pET}-24 b l a_{\mathrm{NDM}-1}$ overproducing NDM-1 enzyme. The checkerboard micro-

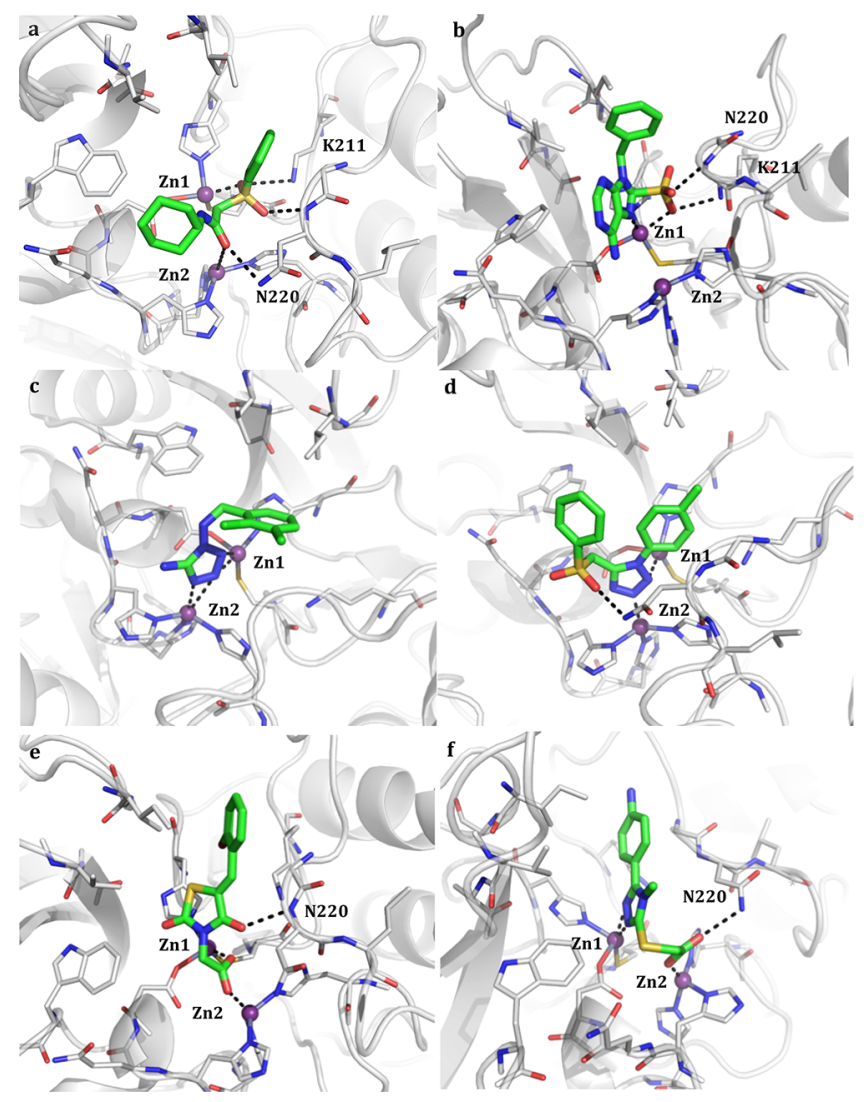

Figure 2. Predicted binding pose of the most representative functionalities in the 31-candidate focused library (Table S1): compounds 3 (a), 7 (b), 8 (c), 11 (d), 12 (e), and 15 (f) in the NDM-1 binding site (Table 1 ). The compounds are shown as capped sticks, zinc ions as purple spheres, and H-bonds as dashed lines. Some residues have been removed for clarity. Pictures were prepared using Pymol (http://www.pymol.org).

dilution assay makes possible to test in a 96-well microplate 77 possible combinations of two drugs simultaneously, allowing the estimation of the fractional inhibitory concentration index (FICI). Since it was not possible to determine the MIC values of the tested NDM-1 inhibitors in the used range (maximum concentration $256 \mu \mathrm{g} / \mathrm{mL}$ ), the FICI index was estimated as the maximum value that can be obtained assigning to the inhibitors a MIC value equal to $512(\mu \mathrm{g} / \mathrm{mL})$. As reported in Tables 2 and 3, compounds 1 and 2 synergically interact with Meropenem, the preferential substrate of NDM-1 enzyme. At a concentration of $128 \mu \mathrm{g} / \mathrm{mL}, 1$ reduces the MIC value of Meropenem in the clinical isolate and the recombinant strain 8 - and 16-fold, respectively.

A 4-fold reduction is observed in both strains when Meropenem is combined with 2 . With ampicillin, cefazolin, and cefotaxime, no synergic activity was observed with the exception of compound $\mathbf{1}$ in combination with cefotaxime in the recombinant strain, where a 4-fold reduction of the antibiotic MIC value is reported. The obtained results show that compounds $\mathbf{1}$ and $\mathbf{2}$ display a significant synergistic effect when in combination with Meropenem, against clinical strain overexpressing NDM-1.

We report the successful application of structure-based virtual screening to the discovery of novel ligands active against NDM-1. Several recurrent chemical classes of MBL inhibitors have been reported over the years, here efficaciously recognized by the 
Table 2. In Vitro Interaction between Antibiotics and Novel NDM-1 Inhibitors Determined by FICI in E. coli BL21 (DE3) pET$24 \mathrm{a}-b l a_{\mathrm{NDM}-1}$

\begin{tabular}{|c|c|c|c|c|c|c|}
\hline \multirow[b]{2}{*}{ inhibitor } & \multicolumn{6}{|c|}{ FIC index ${ }^{a}$} \\
\hline & antibiotic & $\mathrm{MIC}^{b}$ antibiotic $(\mu \mathrm{g} / \mathrm{mL})$ & $\mathrm{MIC}^{b}$ combination $(\mu \mathrm{g} / \mathrm{mL})$ & inhibitor $^{b}$ concentration $(\mu \mathrm{g} / \mathrm{mL})$ & $\Sigma \mathrm{FIC}_{\min }$ & INT \\
\hline \multirow[t]{8}{*}{1} & AMP & 1024 & 1024 & $>256$ & $>1.0$ & IND \\
\hline & & & 0.0625 & 256 & $<0.563$ & IND \\
\hline & MEM & 1 & 0.25 & 16 & $<0.281$ & SYN \\
\hline & & & 0.5 & 0.25 & $<0.501$ & IND \\
\hline & CFZ & 32 & 32 & $>256$ & $>1.0$ & IND \\
\hline & & & 1 & 256 & $<0.625$ & IND \\
\hline & CTX & 8 & 2 & 4 & $<0.258$ & SYN \\
\hline & & & 4 & 0.25 & $<0.505$ & IND \\
\hline \multirow[t]{5}{*}{2} & AMP & 2048 & 1024 & 0.25 & $<0.501$ & IND \\
\hline & MEM & 1 & 0.25 & 64 & $<0.375$ & SYN \\
\hline & & & 0.5 & 0.5 & $<0.501$ & IND \\
\hline & CFZ & $>32$ & 32 & 0.25 & $<0.501$ & IND \\
\hline & CTX & 8 & 4 & 0.25 & $<0.501$ & IND \\
\hline
\end{tabular}

${ }^{a}$ INT, interpretation; IND, indifference; SYN, synergy; ANT, antagonism. Synergy is defined when the FICI is $\leq 0.5$, antagonism when the FICI is $>4$, and indifference when the FICI is $>0.5$ and $\leq 4 .{ }^{b} \mathrm{MIC}$ values were determined as the median of three independent experiments. N.D.: not determined.

Table 3. In Vitro Interaction between Antibiotics and NDM-1 Inhibitors Determined by FICI in E. coli Clinical Strain Overexpressing NDM-1

\begin{tabular}{|c|c|c|c|c|c|c|}
\hline \multirow[b]{2}{*}{ inhibitor } & \multicolumn{6}{|c|}{ FIC index ${ }^{a}$} \\
\hline & antibiotic & $\mathrm{MIC}^{b}$ antibiotic $(\mu \mathrm{g} / \mathrm{mL})$ & MIC $^{b}$ combination $(\mu \mathrm{g} / \mathrm{mL})$ & inhibitor $^{b}$ concentration $(\mu \mathrm{g} / \mathrm{mL})$ & $\Sigma \mathrm{FIC}_{\min }$ & INT \\
\hline \multirow[t]{6}{*}{1} & AMP & $>32768$ & $>32768$ & $>256$ & N.D. & \\
\hline & & & 16 & 128 & $<0.375$ & SYN \\
\hline & MEM & 128 & 32 & 32 & $<0.313$ & SYN \\
\hline & & & 64 & 2 & $<0.504$ & IND \\
\hline & CFZ & 2048 & 2048 & $>256$ & $>1.0$ & IND \\
\hline & CTX & 1024 & 1024 & $>256$ & $>1.0$ & IND \\
\hline \multirow[t]{5}{*}{2} & AMP & $>32768$ & $>32768$ & $>256$ & N.D. & \\
\hline & MEM & 128 & 32 & 32 & $<0.313$ & SYN \\
\hline & & & 64 & 0.5 & $<0.501$ & IND \\
\hline & CFZ & 2048 & 2048 & $>256$ & $>1.0$ & IND \\
\hline & CTX & 1024 & 1024 & $>256$ & $>1.0$ & IND \\
\hline
\end{tabular}

${ }^{a}$ INT, interpretation; IND, indifference; SYN, synergy; ANT, antagonism. Synergy is defined when the FICI is $\leq 0.5$, antagonism when the FICI is $>4$, and indifference when the FICI is $>0.5$ and $\leq 4$. ${ }^{b} \mathrm{MIC}$ values were determined as the median of three independent experiments. N.D.: not determined.

virtual strategy applied: thiols, $N$-arylsulphonyl hydrazones, succinic acids, and mercaptocarboxylate acids were included among our candidates. ${ }^{16-18}$

With respect to other disclosed inhibitors active against NDM1 , undoubtedly, our results stand out for the variety of chemical functionality, correct modeling prediction of the interaction with the crucial $\mathrm{Zn}$ atoms, high complementarity with the target together with drug likeness and ligand efficiency LE (Table S2).

Among the compounds selected by means of SBVS approaches, several demonstrated low micromolar activity toward the target ( $K_{\mathrm{i}}$ for the best selected inhibitor equal to $0.72 \mu \mathrm{M})$. The identified inhibitors all share a common competitive inhibition mechanism vs NDM-1 (Figure S1). In microbiological tests, the synergic activity in association with Meropenem was demonstrated also against clinical resistant strains.

The ability of the best performing compounds to synergize antibiotics against pathogenic resistant bacteria reverting resistance in clinical isolates is encouraging. For the best two compounds, studies for improving their affinity and confirming their binding mode via X-ray crystallography are ongoing.
In the emergence of bacteria resistance new strategies, targets and compounds are urgently needed. Despite the source of structural information on NDM-1 at the atomic level has raised, the challenge to design a valid NDM-1 inhibitor is wide open.

Our results demonstrate the feasibility of applying virtual screening methodologies in identifying novel inhibitors for NDM-1, despite is malleable active site, providing a mechanism base for rational design of NDM-1 inhibitors.

\section{EXPERIMENTAL PROCEDURES}

Structure-Based Virtual Screening. The crystallographic structures of NDM-1 from Klebsiella pneumoniae in the apo form and complexed with ampicillin (PDB codes 3 spu and $3 \mathrm{q} 6 \mathrm{x}$, respectively) were used as templates for SBVS experiments. The binding site was identified and defined by flapsite, implemented in FLAP. ${ }^{13}$ The Specs database (www.specs.net) was chosen as the starting library. This database is part of the ZINC archive (www.zinc.docking.org) and, according to previous experiences, ${ }^{14}$ contains molecules with significant chemical and geometric diversity and good purity and availability. A set of about 300,000 compounds was downloaded and filtered according to $\log \mathrm{P}$ values calculated by Moka. ${ }^{25}$ In order to ensure sufficient solubility, only molecules with $\log \mathrm{P}<2.5$ were retained, amounting, in 
this experiment, to 22,634. Tautomers and protomers were added. The virtual screening was performed with FLAPdock implemented within FLAP software, developed and licensed by Molecular Discovery Ltd. (www.moldiscovery.com). FLAP is based on the Molecular Interaction Fields (MIFs) calculated by GRID. ${ }^{26}$ A specific version of FLAPdock was developed to deal with the MBL active site. The native docking scoring function was modified to add a classical electrostatic term and also a soft Lennard-Jones term. These terms were scaled for comparison with the FLAPdock MIF similarity score. Additional MIFs for the DRY, $\mathrm{O}$, and $\mathrm{N} 1$ fields were also added, but cut at the most favorable $10 \%$ to emphasize the strongest hotspots in the site. The modified docking algorithm was validated on the DUD data set. ${ }^{27}$ Molecules were ranked according to the FLAP-S Score function, containing four terms to evaluate the similarity of the $\mathrm{H}$ (shape), $\mathrm{O}$ (H-bond acceptor), $\mathrm{N} 1$ ( $\mathrm{H}$ bond donor), and DRY (Hydrophobic) MIFs between the template pocket on NDM-1 and the candidates, a Lennard-Jones potential, an electrostatic term, and a penalization term to penalize $\mathrm{H}$-bond donor groups in H-bond acceptor MIFs, and vice versa. After the first VS, the highest score compounds ( $\sim 1 \%$ of the initial library) were redocked in the binding site with a more accurate modality, taking more computational time but providing more reliable results. All compounds in the pocket were visually inspected, and on the basis of (i) the FLAP Sscore, the superposition with the pocket's MIFs, (ii) the interactions with the surrounding residues, and (iii) the chemical diversity, 100 compounds were selected for testing.

Protein Sovra Expression and Purification. E. coli JM109(DE3) cells containing the recombinant plasmids $\mathrm{pFM}-\mathrm{NDM}-1$ were grown in $1 \mathrm{~L}$ of tryptic soy broth (TSB) medium with kanamycin $(50 \mu \mathrm{g} / \mathrm{mL})$ at $37^{\circ} \mathrm{C}$ in an orbital shaker (180 rpm). Each culture was grown to achieve an $A 600$ of approximately 0.5 , and $0.4 \mathrm{mM}$ IPTG (isopropyl- $\beta$-Dthiogalac-topyranoside) was added. After the addition of IPTG, the cultures were incubated for $16 \mathrm{~h}$ at $22{ }^{\circ} \mathrm{C}$, under aerobic conditions. Cells were harvested by centrifugation at $8000 \mathrm{rpm}$ for $10 \mathrm{~min}$ at $4{ }^{\circ} \mathrm{C}$ and washed twice with $20 \mathrm{mM}$ Tris- $\mathrm{HCl}$ buffer ( $\mathrm{pH}$ 7.0) (buffer A). Crude enzymes were obtained by treatment with lysozyme at a concentration of $2 \mathrm{mg} / \mathrm{mL}$ for $30 \mathrm{~min}$ at $30^{\circ} \mathrm{C}$ followed by sonication on ice (five cycles at $60 \mathrm{~W}$ for $1 \mathrm{~min}$ and $2 \mathrm{~min}$ of break). The lysate was centrifuged at $30000 \mathrm{rpm}$ for $30 \mathrm{~min}$, and the cleared supernatant was recovered and loaded onto a Sepharose- $\mathrm{Q}$ fast-flow column equilibrated with buffer $A$. The column was extensively washed to remove unbound proteins, and the $\beta$-lactamase was eluted with a linear gradient of $20 \mathrm{mM}$ Tris- $\mathrm{HCl}(\mathrm{pH} 7.0)-\mathrm{NaCl}(0.5 \mathrm{M})$ (buffer B). The fractions containing $\beta$-lactamase activity were pooled, concentrated 20 -fold using an Amicon concentrator (YM 10 membrane; Millipore, Bedford, MA, USA), and loaded onto Sephacryl S-100 equilibrated with buffer A. The pure fractions were pooled and dialyzed in $20 \mathrm{mM}$ HEPES buffer ( $\mathrm{pH} 7.0)$ containing $0.01 \mathrm{mg} / \mathrm{mL}$ bovine serum albumin (BSA) (buffer C) for further experiments. ${ }^{28}$

Inhibition Assays. All molecules (from Specs; www.specs.net, tested without further purification; compounds purity was always above $90 \%$ as declared by Specs) were tested toward NDM-1 enzyme purified at homogeneity as previously described. ${ }^{28}$ For kinetic experiments the molecules were dissolved in $100 \%$ DMSO to have a final concentration in cuvettes of $1 \%$. The inhibition assays were carried out by preincubating, for $10 \mathrm{~min}$ at $25{ }^{\circ} \mathrm{C}, 0.5 \mathrm{nM}$ pure enzyme with various concentrations of inhibitors (from 0 to $500 \mu \mathrm{M}$ ) in $20 \mathrm{mM}$ HEPES buffer ( $\mathrm{pH} 7.0$ ) containing $20 \mu \mathrm{M} \mathrm{ZnCl}_{2}$. The rate of inhibition was measured by the reporter substrate method ${ }^{15}$ by using $30 \mu \mathrm{M}$ nitrocefin at the following conditions:

$$
v_{0} / v_{\mathrm{i}}=1+\left(K_{\mathrm{m}} I\right) /\left(K_{\mathrm{m}}+S\right) K_{\mathrm{i}}
$$

where $v_{i}$ and $v_{0}$ represent the initial rates of hydrolysis of nitrocefin with or without inhibitor, respectively; $I$ is the concentration of inhibitor, $K_{\mathrm{i}}$ is the inhibition constant, $K_{\mathrm{m}}$ is the Henri-Michaelis constant, and $S$ is the concentration of the reporter substrate. The plot $v_{0} / v_{\mathrm{i}}$ versus $[I]$ yielded a straight line of slope $K_{\mathrm{m}} /\left(K_{\mathrm{m}}+S\right) K_{\mathrm{i} .}^{15}$ The data were collected with a Lambda 25 spectrophotometer (PerkinElmer Italia).

Checkerboard Microdilution Assay. The in vitro interactions between ampicillin, Meropenem (MEM), cefazolin (CFZ), and cefotaxime (CTX) and best inhibitors 1 and $\mathbf{2}$ were investigated by a two-dimensional checkerboard microdilution assay, using 96-well microtitration plates as previously described. ${ }^{29}$ The microtiter plates were incubated at $37^{\circ} \mathrm{C}$ for $18 \mathrm{~h}$. The growth in each well was quantified spectrophotometrically at $595 \mathrm{~nm}$ by a microplate reader iMark, BioRad (Milan, Italy). The percentage of growth in each well was calculated as

$$
\frac{O D_{\text {drug combination well }}-O D_{\text {background }}}{O D_{\text {drug-free well }}-O D_{\text {background }}}
$$

where the background was obtained from the microorganism-free plates, processed as the inoculated plates. The MIC for each combination of drugs was defined as the concentration of drug that reduced bacterial growth by $80 \%$ compared to that of organisms grown in the absence of drug. All experiments were performed in triplicate.

Drug Interaction Models. In order to assess the nature of the in vitro interactions between the compounds and antibiotics against the recombinant E. coli BL21 (DE3) pET-24a-bla $a_{\mathrm{NDM}-1}$ strain and against the E. coli clinical strain overexpressing NDM-1, the data obtained from the checkerboard assay were analyzed to calculate the fractional inhibitory concentration index. ${ }^{30}$ The fractional inhibitory concentration index (FICI) is the mathematical expression of the effect of the combination of antibacterial agents expressed as

$$
\Sigma \mathrm{FIC}=\mathrm{FIC}_{\mathrm{A}}+\mathrm{FIC}_{\mathrm{B}}=\frac{\mathrm{MIC}_{\mathrm{AB}}}{\mathrm{MIC}_{\mathrm{A}}}+\frac{\mathrm{MIC}_{\mathrm{BA}}}{\mathrm{MIC}_{\mathrm{B}}}
$$

where $\mathrm{MIC}_{\mathrm{A}}$ and $\mathrm{MIC}_{\mathrm{B}}$ are the MICs of drugs $\mathrm{A}$ and $\mathrm{B}$ when acting alone and $\mathrm{MIC}_{\mathrm{AB}}$ and $\mathrm{MIC}_{\mathrm{BA}}$ are the MICs of drugs $\mathrm{A}$ and $\mathrm{B}$ when acting in combination. Among all $\Sigma F I C s$ calculated for each microplate, the FICI was determined as the lowest $\Sigma \mathrm{FIC}\left(\Sigma \mathrm{FIC} C_{\text {min }}\right)$ when synergy is supposed, or the highest $\Sigma \mathrm{FIC}\left(\Sigma \mathrm{FIC}_{\max }\right)$ when antagonism is evident. Since in MIC determination the variation in a single result places a MIC value in a three-dilution range $( \pm 1$ dilution), therefore, the reproducibility errors in the MIC checkerboard assays are considerable. For that reason, the interpretation of FICI data should be done taking into consideration values well below or above the theoretical cutoff (1.0) defined by Berenbaum. Synergy was, therefore, defined when FICI $\leq$ 0.5 , while antagonism was defined when FICI $>4$. A FIC index between 0.5 and $4(0.5<$ FICI $\leq 4)$ was considered indifferent. ${ }^{31}$

\section{ASSOCIATED CONTENT}

\section{Supporting Information}

The Supporting Information is available free of charge on the ACS Publications website at DOI: 10.1021/acsmedchemlett.7b00428.

Focused library: 31 candidates selected for further validation vs NDM-1 in Table S1. In silico properties and ligand efficiencies for candidates in Table S2. Kinetic characterization of NDM-1 inhibition by compounds 1 and $\mathbf{2}$ in Figure S1. SDS-PAGE gel of purified NDM-1 in Figure S2. (PDF)

\section{AUTHOR INFORMATION}

\section{Corresponding Author}

*Phone: +39 059205 8577. E-mail: tondid@unimore.it. ORCID $\odot$

Francesca Spyrakis: 0000-0002-4016-227X

Donatella Tondi: 0000-0002-5195-5531

\section{Present Address}

${ }^{\perp}$ Dipartimento di Scienza e Tecnologia del Farmaco, Università degli Studi di Torino, Via Pietro Giuria 9, 10125 Torino, Italy.

\section{Author Contributions}

The manuscript was written through contributions of all authors and all have given approval to the final version of the manuscript. 


\section{Funding}

This work was supported by Finanziamento per la Ricerca di Ateneo (FAR 2014), Università degli Studi di Modena e Reggio Emilia to D.T.

\section{Notes}

The authors declare no competing financial interest.

\section{ABBREVIATIONS}

NDM-1, New Delhi Metallo- $\beta$-lactamase-1; ESBLs, extended spectrum $\beta$-lactamases; SBLs, serine based BLs; MBLs, metallo$\beta$-lactamases; FLAP, Fingerprints for Ligands and Proteins; FICI, fractional inhibitory concentration index; MIC, minimum inhibitory concentration; LE, ligand efficiency

\section{REFERENCES}

(1) WHO. Antimicrobial Resistance: Global Report on Surveillance, 2014. http://apps.who.int/iris/bitstream/10665/112642/1/ 9789241564748 eng.pdf.

(2) Frere, J. M.; Sauvage, E.; Kerff, F. From "An Enzyme Able to Destroy Penicillin" to Carbapenemases: 70 Years of Beta-lactamase Misbehaviour. Curr. Drug Targets 2016, 17 (9), 974-82.

(3) Tondi, D.; Cross, S.; Venturelli, A.; Costi, M. P.; Cruciani, G.; Spyrakis, F. Decoding the Structural Basis For Carbapenem Hydrolysis By Class A beta-lactamases: Fishing For A Pharmacophore. Curr. Drug Targets 2016, 17 (9), 983-1005.

(4) Tondi, D.; Venturelli, A.; Bonnet, R.; Pozzi, C.; Shoichet, B. K.; Costi, M. P. Targeting class A and C serine beta-lactamases with a broadspectrum boronic acid derivative. J. Med. Chem. 2014, 57 (12), 544958.

(5) Mojica, M. F.; Bonomo, R. A.; Fast, W. B1-Metallo-betaLactamases: Where Do We Stand? Curr. Drug Targets 2016, 17 (9), 1029-50.

(6) Ambler, R. P. The structure of beta-lactamases. Philos. Trans. $R$. Soc., B 1980, 289 (1036), 321-31.

(7) Farina, D.; Spyrakis, F.; Venturelli, A.; Cross, S.; Tondi, D.; Costi, M. P. The inhibition of extended spectrum beta-lactamases: hits and leads. Curr. Med. Chem. 2014, 21 (12), 1405-34.

(8) Bush, K.; Page, M. G. P. What we may expect from novel antibacterial agents in the pipeline with respect to resistance and pharmacodynamic principles. J. Pharmacokinet. Pharmacodyn. 2017, 44, $113-132$.

(9) Walsh, T. R.; Toleman, M. A.; Poirel, L.; Nordmann, P. Metallobeta-lactamases: the quiet before the storm? Clin Microbiol Rev. 2005, 18 (2), 306-25.

(10) Fair, R. J.; Tor, Y. Antibiotics and bacterial resistance in the 21st century. Perspect. Med. Chem. 2014, 6, 25-64.

(11) Poirel, L.; Lagrutta, E.; Taylor, P.; Pham, J.; Nordmann, P. Emergence of metallo-beta-lactamase NDM-1-producing multidrugresistant Escherichia coli in Australia. Antimicrob. Agents Chemother. 2010, 54 (11), 4914-6.

(12) Nordmann, P.; Poirel, L.; Toleman, M. A.; Walsh, T. R. Does broad-spectrum beta-lactam resistance due to NDM-1 herald the end of the antibiotic era for treatment of infections caused by Gram-negative bacteria? J. Antimicrob. Chemother. 2011, 66 (4), 689-92.

(13) Baroni, M.; Cruciani, G.; Sciabola, S.; Perruccio, F.; Mason, J. S. A common reference framework for analyzing/comparing proteins and ligands. Fingerprints for Ligands and Proteins (FLAP): theory and application. J. Chem. Inf. Model. 2007, 47 (2), 279-94.

(14) Spyrakis, F.; Cellini, B.; Bruno, S.; Benedetti, P.; Carosati, E.; Cruciani, G.; Micheli, F.; Felici, A.; Cozzini, P.; Kellogg, G. E.; Voltattorni, C. B.; Mozzarelli, A. Targeting cystalysin, a virulence factor of treponema denticola-supported periodontitis. ChemMedChem 2014, 9 (7), 1501-11.

(15) De Meester, F.; Joris, B.; Reckinger, G.; Bellefroid-Bourguignon, C.; Frere, J. M.; Waley, S. G. Automated analysis of enzyme inactivation phenomena. Application to $\beta$-lactamases and DD-peptidases. Biochem. Pharmacol. 1987, 36, 2393-2403.
(16) Chen, A. Y.; Thomas, P. W.; Stewart, A. C.; Bergstrom, A.; Cheng, Z.; Miller, C.; Bethel, C. R.; Marshall, S. H.; Credille, C. V.; Riley, C. L.; Page, R. C.; Bonomo, R. A.; Crowder, M. W.; Tierney, D. L.; Fast, W.; Cohen, S. M. Dipicolinic Acid Derivatives as Inhibitors of New Delhi Metallo-beta-lactamase-1. J. Med. Chem. 2017, 60 (17), 7267-7283.

(17) Klingler, F. M.; Wichelhaus, T. A.; Frank, D.; Cuesta-Bernal, J.; ElDelik, J.; Muller, H. F.; Sjuts, H.; Gottig, S.; Koenigs, A.; Pos, K. M.; Pogoryelov, D.; Proschak, E. Approved Drugs Containing Thiols as Inhibitors of Metallo-beta-lactamases: Strategy To Combat MultidrugResistant Bacteria. J. Med. Chem. 2015, 58 (8), 3626-30.

(18) Groundwater, P. W.; Xu, S.; Lai, F.; Varadi, L.; Tan, J.; Perry, J. D.; Hibbs, D. E. New Delhi metallo-beta-lactamase-1: structure, inhibitors and detection of producers. Future Med. Chem. 2016, 8 (9), 993-1012.

(19) Sevaille, L.; Gavara, L.; Bebrone, C.; De Luca, F.; Nauton, L.; Achard, M.; Mercuri, P.; Tanfoni, S.; Borgianni, L.; Guyon, C.; Lonjon, P.; Turan-Zitouni, G.; Dzieciolowski, J.; Becker, K.; Benard, L.; Condon, C.; Maillard, L.; Martinez, J.; Frere, J. M.; Dideberg, O.; Galleni, M.; Docquier, J. D.; Hernandez, J. F. 1,2,4-Triazole-3-thione Compounds as Inhibitors of Dizinc Metallo-beta-lactamases. ChemMedChem 2017, 12 (12), 972-985.

(20) Nauton, L.; Kahn, R.; Garau, G.; Hernandez, J. F.; Dideberg, O. Structural insights into the design of inhibitors for the L1 metallo-betalactamase from Stenotrophomonas maltophilia. J. Mol. Biol. 2008, 375 (1), 257-69.

(21) Jin, W.; Arakawa, Y.; Yasuzawa, H.; Taki, T.; Hashiguchi, R.; Mitsutani, K.; Shoga, A.; Yamaguchi, Y.; Kurosaki, H.; Shibata, N.; Ohta, M.; Goto, M. Comparative study of the inhibition of metallo-betalactamases (IMP-1 and VIM-2) by thiol compounds that contain a hydrophobic group. Biol. Pharm. Bull. 2004, 27 (6), 851-6.

(22) King, D. T.; Worrall, L. J.; Gruninger, R.; Strynadka, N. C. New Delhi metallo-beta-lactamase: structural insights into beta-lactam recognition and inhibition. J. Am. Chem. Soc. 2012, 134 (28), 11362-5.

(23) Antony, J.; Gresh, N.; Olsen, L.; Hemmingsen, L.; Schofield, C. J.; Bauer, R. Binding of D- and L-captopril inhibitors to metallo-betalactamase studied by polarizable molecular mechanics and quantum mechanics. J. Comput. Chem. 2002, 23 (13), 1281-96.

(24) Hopkins, A. L.; Keseru, G. M.; Leeson, P. D.; Rees, D. C.; Reynolds, C. H. The role of ligand efficiency metrics in drug discovery. Nat. Rev. Drug Discovery 2014, 13 (2), 105-21.

(25) Milletti, F.; Storchi, L.; Goracci, L.; Bendels, S.; Wagner, B.; Kansy, M.; Cruciani, G. Extending pKa prediction accuracy: highthroughput $\mathrm{pKa}$ measurements to understand $\mathrm{pKa}$ modulation of new chemical series. Eur. J. Med. Chem. 2010, 45 (9), 4270-9.

(26) Wade, R. C.; Goodford, P. J. The role of hydrogen-bonds in drug binding. Prog. Clin Biol. Res. 1989, 289, 433-44.

(27) Huang, N.; Shoichet, B. K.; Irwin, J. J. Benchmarking sets for molecular docking. J. Med. Chem. 2006, 49 (23), 6789-801.

(28) Marcoccia, F.; Bottoni, C.; Sabatini, A.; Colapietro, M.; Mercuri, P. S.; Galleni, M.; Kerff, F.; Matagne, A.; Celenza, G.; Amicosante, G.; Perilli, M. Kinetic Study of Laboratory Mutants of NDM-1 Metallobeta-Lactamase and the Importance of an Isoleucine at Position 35. Antimicrob. Agents Chemother. 2016, 60 (4), 2366-72.

(29) Bellio, P.; Segatore, B.; Mancini, A.; Di Pietro, L.; Bottoni, C.; Sabatini, A.; Brisdelli, F.; Piovano, M.; Nicoletti, M.; Amicosante, G.; Perilli, M.; Celenza, G. Interaction between lichen secondary metabolites and antibiotics against clinical isolates methicillin-resistant Staphylococcus aureus strains. Phytomedicine 2015, 22 (2), 223-30.

(30) Greco, W. R.; Bravo, G.; Parsons, J. C. The search for synergy: a critical review from a response surface perspective. Pharmacol Rev. 1995, 47 (2), 331-85.

(31) Odds, F. C. Synergy, antagonism, and what the chequerboard puts between them. J. Antimicrob. Chemother. 2003, 52 (1), 1. 